\title{
A New Technique for ARMA-System Identification Based on QR-Decomposition of Third Order Cumulants Matrix
}

\author{
Adnan M Al-Smadi \\ Department of Electronics Engineering, Yarmouk University, \\ Irbid, Jordan
}

Received: April 27, 2021. Received: October 4, 2021. Accepted: October 21, 2021. Published: November 1, 2021.

\begin{abstract}
In this paper a new technique to estimate the coefficients of a general Autoregressive Moving Average (ARMA) $(p, q)$ model is proposed. The ARMA system is excited by an un-observable independently identically distributed (i.i.d) non-Gaussian process. The proposed ARMA coefficients estimation method uses the QR-Decomposition (QRD) of a special matrix built with entries of third order cumulants (TOC) of the available output data only. The observed output may be corrupted with additive colored or white Gaussian noise of unknown power spectral density. The proposed technique was compared with several good methods such as the residual time series (RTS) and the Q-slice algorithm (QSA) methods. Simulations for several examples were tested. The results for these examples confirm the good performance of the proposed technique with respect to existing well-known methods.
\end{abstract}

Keywords - Time series Analysis, higher order statistics, signal-to-noise-ratio, Gaussian processes.

\section{INTRODUCTION}

$\mathrm{T}_{\mathrm{a}}^{\mathrm{H}}$ HE Autoregressive moving average (ARMA) models play a significant role in signal restoration, predicting time series data, and system identification [1]. The problem of estimating the coefficients of ARMA models has attracted much attention because of its broad applications to many fields such as adaptive control, signal modeling, and biomedical signal processing. The literature has many contributions on estimating the ARMA process coefficients using second order and higher order cumulants [2, 3, 4, 5]. Giannakis and Mendel [2] developed a Residual Time Series (RTS) algorithm for the identification linear time invariant (LTI) nonminimum phase systems when only output data are available. The authors assumed that the order is given in modeling an autoregressive moving-average process. The basic idea of the algorithms in the literature is to estimate the AR coefficient. Then, a residual
MA time series is formed. Finally, the MA coefficients are estimated. Swami and Mendel [3] developed a Q-slice algorithm (QSA) for estimating the ARMA coefficients that uses q 1-D slices of the output cumulants. This method does not involve any computations of the residuals.

The second order statistics (SOS) algorithms work smooth if the signal has Gaussian distribution [6]. Nevertheless, there are many realistic signals are non-Gaussian. Hence, in practice, there are situations where we must look beyond the autocorrelation (second order cumulants) of the available data.

Signal processing with higher order statistics (or cumulants), has been studied in the literature [1-4, 7]. Cumulants have gained a lot of interest in signal processing for many reasons. One of the main reasons for this interest is that third order cumulants (and higher orders) are zeros. Hence, if the present signal in non-Gaussian with Gaussian additive noise, the noise will disappear in the third order cumulant (and higher) [8].

The $\mathrm{QR}$ decomposition is a significant operation in solving many linear algebra problems [9]. The idea of the QR decomposition is to factor a given matrix into two parts: $\mathrm{Q}$ and $\mathrm{R}$ in which the first one is orthogonal matrix and the second is upper right triangular matrix (with nonzero diagonal elements). Poomvichid, Patirupanusara, and Ketcham [10] presented a procedure to hide data in audio watermark systems based on QR decomposition and Genetic Algorithms (GA) using the discrete wavelet transform. Wang, Liu, and Zhu [11] proposed a blind water-marking method based on QR factorization and color image using discrete wavelet transform. Amiri and Fathy [9] presented an approach for shot boundary detection using QR-decomposition

In system identification, QR-decomposition method has been used often to solve the least squares problems and generalized linear regression problems in engineering and science problems [12]. Mehena, Swain, and Patnaik [13] proposed a $\mathrm{QR}$ factorization rotation-based recursive least squares algorithm. They derived the QR-RLS method using a single matrix to solve different least squares problems. Pickhardt and Unbehauen [14] presented a numerically robust multi-step identification scheme using QR-decomposition for the identification of single-input single-output systems. 
This paper presents an approach to estimate the coefficients of non-Gaussian $\operatorname{ARMA}(p, q)$ process in additive colored (or white) Gaussian noise. The proposed method is based on the QR-Decomposition (QRD) of a special matrix with entries of the third order cumulants (TOC) of the available output data only. The proposed algorithm will abbreviated as QRD-TOC. The paper is organized as follows. Section 2 considers some preliminaries about ARMA $(p, q)$ model and assumptions. Problem Formulation is presented in Section 3. Results and discussion are given in Section 4. Concluding remarks are presented in Section 5.

\section{PRELIMINARIES}

The observed times series is modeled as the output of an Autoregressive Moving Average (ARMA) $(p, q)$ process that is excited by an unobservable input, and is corrupted at its output by additive colored Gaussian noise. The time series is described by

$$
\begin{aligned}
& \sum_{k=0}^{p} a(k) y(n-k)=\sum_{k=0}^{q} b(k) u(n-k) \\
& s(n)=y(n)+w(n)
\end{aligned}
$$

where $u(n)$ is the system input, $y(n)$ is the noiseless system output, $w(n)$ is the additive Gaussian noise, and $s(n)$ is the observed noisy time series.

The system in (1) produces an ARMA $(p, q)$ process. The $a_{i}$ 's are the autoregressive (AR) parameters and the $b_{i}$ 's are the moving average (MA) parameters; $p$ is the order of AR part whereas $q$ is the order of the MA part. The following assumptions are made:

1) The model order $(p, q)$ are known.

2) The input $u(n)$ is unobservable independently identically distributed (i.i.d) non-Gaussian process.

3) The additive noise, $w(n)$, is independent of the input, $u(n)$, and hence, of the output, $y(n)$.

The Z-transfer for the system in (1) is

$$
Y(z) \sum_{k=0}^{q} b_{k} z^{-k}=U(z) \sum_{k=0}^{p} a_{k} z^{-k}
$$

The system function is

$$
H(z)=\frac{Y(z)}{U(z)}=\frac{\sum_{k=0}^{q} b_{k} z^{-k}}{1+\sum_{k=1}^{p} a_{k} z^{-k}}=\frac{B(Z)}{A(Z)}
$$

The frequency response of the filter, $H(f)$, is obtained at $z=e^{j w T}$ in (4)

$$
H(f)=\frac{\sum_{k=0}^{q} b_{k} e^{-j k w T}}{1+\sum_{k=1}^{p} a_{k} e^{-j k w T}}
$$

where $T$ is the sampling period and $w$ is the angular frequency. The problem under study is to estimate the ARMA coefficients $a_{i}$ 's and $b_{i}$ 's from noisy observation $s(k)$.

\section{PROBLEM FORMULATION}

Let the system output $y(k)$ be zero-mean stationary ergodic random process. Then, the third-order cumulant of $y(k)$ is given by

$$
C_{3 y}(n, m)=E[y(k) y(k+n) y(k+m)]
$$

The cross-cumulant between the input and output sequences, $u(k)$ and $y(k)$ respectively, is given by

$$
C_{u y y}(n, m)=E[u(k) y(k+n) y(k+m)]
$$

The sequence $y(k)$ is observed in the noisy signal $s(k)$ as in (2). Multiplying both sides of (2) by $s(k+n) s(k+m)$,

$$
\begin{aligned}
& \quad s(k) s(k+n) s(k+m)= \\
& y(k) s(k+n) s(k+m)+w(k) s(k+n) s(k+m) \\
& \quad=y(k)[y(k+n)+w(k+n)][y(k+m)+w(k+m)]+ \\
& w(k) s(k+n) s(k+m)
\end{aligned}
$$

Simplifying (8) and taking the expected value $E[s(k) s(k+n) s(k+m)]=E[y(k) y(k+n) y(k+m)]+$ $E[y(k) y(k+n) w(k+m)]+E[y(k) y(k+m) w(k+n)]+$

$E[y(k) w(k+n) w(k+m)]+E[w(k) s(k+n) s(k+m)]$

It can be seen that these terms are cumulants and crosscumulants as defined in (6) and (7). Since the additive noise is independent of $y(k)$ and $s(k)$, then the second, the third, and the fourth terms of the right hand side of (9) become zeros. Hence, the cumulants of the noisy output are theoretically identical to the noiseless output sequence. That is

$$
E[s(k) s(k+n) s(k+m)]=E[y(k) y(k+n) y(k+m)]
$$

or

$$
C_{3 s}=C_{3 y}
$$

Now, multiply both sides of (1) by $y(k+n) y(k+m)$

$a_{0} y(k) y(k+n) y(k+m)+$

$a_{1} y(k-1) y(k+n) y(k+m)+\cdots+$

$a_{p} y(k-p) y(k+n) y(k+m)=$

$b_{0} u(k) y(k+n) y(k+m)+$

$b_{1} u(k-1) y(k+n) y(k+m)+\cdots+$

$b_{q} u(k-q) y(k+n) y(k+m)$

The expected value for (11),

$$
\begin{aligned}
& a_{0} C_{3 y}(n, m)+a_{1} C_{3 y}(n+1, m+1) \\
& +\cdots+a_{p} C_{3 y}(n+p, m+p)=
\end{aligned}
$$




$$
\begin{aligned}
& b_{0} C_{u y y}(n, m)+b_{1} C_{u y y}(n+1, m+1) \\
& +\cdots+b_{q} C_{u y y}(n+q, m+q)
\end{aligned}
$$

By stacking (12) for several values of $n$ and $m$ ranging from $\psi$ to $\psi$ where $\psi$ denotes the range of the third order cumulants to be used, the system in (12) can be expressed in matrix format as follows

$$
\underline{f}=-C_{3 y} \underline{a}_{p}+C_{u y y} \underline{b}_{q}
$$

The cumulants at 0 lag (i.e., $n=m=0$ ), are contained in the vector $\underline{f}$. The coefficients of the ARMA $(p, q)$ process are contained in the vectors $\underline{a}_{p}$ and $\underline{b}_{q}$. in (1). The entries of the matrix $C_{3 y}$ represent the cumulants of the output sequence whereas the entries of the matrix $C_{u y y}$ represent the crosscumulants of the input and output data sequences.

Equation (13) can be written as

$$
\underline{f}=C_{p q} \underline{\theta}
$$

where $C_{p q}$ is a composite data matrix contains the cumulants of the output sequence and the cross-cumulants of the input and output sequences

$$
C_{p q}=\left[\begin{array}{ll}
C_{3 y} & C_{u y y}
\end{array}\right]
$$

and $\underline{\theta}$ is the ARMA coefficients vector

$$
\underline{\theta}=\left[\begin{array}{ll}
-\underline{a} & \underline{b}
\end{array}\right]^{T}
$$

Notice that the columns in the linear statistical model in (14) are linearly independent. Hence, we can multiply both sides of (14) by $\left(C_{p q}\right)^{T}$, then

$$
\left(C_{p q}\right)^{T} \underline{f}=\left(C_{p q}\right)^{T} C_{p q} \underline{\theta}
$$

or

$$
\left(C_{p q}\right)^{T} C_{p q} \underline{\theta}=\left(C_{p q}\right)^{T} \underline{f}
$$

The Gram matrix for the data matrix $C_{p q}$ is

$$
G=\left(C_{p q}\right)^{T} C_{p q}
$$

Substituting (19) into (18) yields

$$
G \underline{\theta}=\left(C_{p q}\right)^{T} \underline{f}
$$

or

$$
G \underline{\theta}=\underline{f_{c}}
$$

where $\underline{f_{C}}=\left(C_{p q}\right)^{T} \underline{f}$.

Now, a general matrix $G(m \times n)$ can be decomposed as [15]

$$
G=Q R
$$

where $Q(m \times m)$ is a unitary matrix and $R(m \times n)$ is an upper triangular matrix. If $G$ is square, and the elements of $Q$ being complex, then $Q$ is unitary, $\left(Q^{H}=Q^{-1}\right)$ [16], and

$$
Q^{H} Q=I
$$

The notation $=()^{H}$ denotes the complex conjugate transpose; i.e., $\left(Q^{T}\right)^{*}$ as $(Q)^{H}, Q^{-1}$ is the matrix inverse, and $I$ is the identity matrix. If $Q$ has real elements and

$$
Q^{T} Q=I
$$

then $Q$ is said to be an orthogonal matrix. Substituting (22) into (21), we obtain

$$
(Q R) \underline{\theta}=\underline{f_{C}}
$$

Multiplying both sides of (25) by $(Q R)^{T}$ yields,

$$
(Q R)^{T}(Q R) \underline{\theta}=(Q R)^{T} \underline{f_{\mathcal{C}}}
$$

Simplifying (26),

$$
\left[R^{T} Q^{T} Q R\right] \underline{\theta}=R^{T} Q^{T} \underline{f_{\mathcal{C}}}
$$

Using (24), i.e., ) $Q^{T} Q=I$ ), then

$$
\left[R^{T} R\right] \underline{\theta}=R^{T} Q^{T} \underline{f_{\mathcal{C}}}
$$

Multiply both sides of (28) by $\left[R^{T} R\right]^{-1}$,

$$
\left[R^{T} R\right]^{-1}\left[R^{T} R\right] \underline{\theta}=\left[R^{T} R\right]^{-1} R^{T} Q^{T} \underline{f_{c}}
$$

Simplifying (29),

$$
\underline{\theta}=R^{-1}\left[R^{-T} R^{T}\right] Q^{T} \underline{f_{\mathcal{C}}}
$$

or

$$
\underline{\theta}=R^{-1} Q^{T} \underline{f_{C}}
$$

\section{A. Computing the QR-Decomposition}

The matrix $G$ may be written as the product of an orthogonal matrix, $Q$, and an upper triangular matrix, $R$ as follows:

$$
G=\left[\underline{g}_{1} \underline{g}_{2} \cdots \underline{g}_{n}\right]=
$$

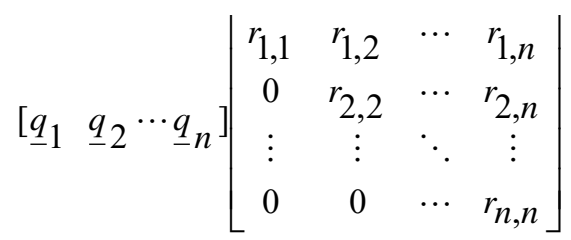

The vectors $\left[\underline{g}_{1} \underline{g}_{2} \cdots \underline{g}_{n}\right]$ are linearly independent. The vectors $\left[\underline{q}_{1} \underline{q}_{2} \cdots \underline{q}_{n}\right]$ are orthonormal vectors; that is,

$$
\left\|\underline{q}_{i}\right\|=1 ; \underline{q}_{i}^{T} \underline{q}_{j}=0 \quad \text { if } i \neq j
$$


Since $R$ has nonzero diagonal elements, then it is nonsingular matrix. This decomposition represents the $n^{\text {th }}$ column of $G$ by linear combination of orthogonal columns:

$$
\underline{g}_{n}=\sum_{k=1}^{n} \underline{q}_{k} r_{k n}
$$

One of the methods to compute the QR decomposition is the Gram-Schmidt and Householder algorithms [17].

The Gram-Schmidt algorithm begins as follows:

$$
\begin{gathered}
\underline{x}_{1}=\underline{g}_{1} ; \\
\underline{q}_{1}=\frac{x_{1}}{\left\|x_{1}\right\|}
\end{gathered}
$$

Now, we construct $x_{2}$ as follows:

$$
\begin{gathered}
\underline{x}_{2}=\underline{g}_{2}-\frac{\underline{g}_{2} \underline{x}_{1}}{\left\|\underline{x}_{1}\right\|^{2}} \underline{x}_{1}=\underline{g}_{2}-\left(\underline{g}_{2}^{T} \bullet \underline{q}_{1}\right) \underline{q}_{1} ; \\
\underline{q}_{2}=\frac{\underline{x}_{2}}{\left\|\underline{x}_{2}\right\|}
\end{gathered}
$$

Continuing this procedure, each new vector $\underline{x}_{k}$ is generated as follows:

$$
\begin{gathered}
\underline{x}_{k}=\underline{g}_{k}-\sum_{i=1}^{k-1}\left(\underline{g}_{k}^{T} \bullet \underline{q}_{i}\right) \underline{q}_{i} ; \\
\underline{q}_{k}=\frac{\underline{x}_{k}}{\left\|\underline{x}_{k}\right\|}
\end{gathered}
$$

Note that $\|x\|$ is the $L_{2}$ norm.

The elements for the $R$ matrix are computed as follows:

$$
\begin{aligned}
r_{1, k} & =\underline{q}_{1} \bullet \underline{g}_{k} \\
r_{2, k} & =\underline{q}_{2} \bullet \underline{g}_{k} \\
\vdots & \\
r_{k-1, k} & =\underline{q}_{k-1} \bullet \underline{g}_{k}
\end{aligned}
$$

\section{B. $\operatorname{ARMA}(p, q)$ Modeling}

Now, the only observed data is the output sequence, $y(n)$. However, the excitation sequence, $u(n)$, is necessary to calculate the cross-cumulants, $C_{u y y}$. Therefore, an estimate for the excitation sequence, $u(n)$, was obtained using the methods in $[18,19]$. The method models the observed output data by a high order AR process. Thus, the system in (1) could be rewritten as

$$
\hat{u}(n)=\sum_{i=0}^{M} \eta_{i} y(n-i)
$$

where $\eta_{i}$ are the coefficients of the high AR process and are estimated as follows.

$\eta=\left[\frac{1}{N+1} \sum_{k=0}^{N} \Phi(k) \underline{\Phi}(k)^{T}\right]^{-1}\left[\frac{1}{N+1} \sum_{k=0}^{N} \underline{\Phi}(k) y(k)\right]$ with

$$
\underline{\Phi}(k)=[-y(k-1)-y(k-2)-\ldots-y(k-J)]^{T}
$$

and $J$ is the order of the high AR process. Using $\hat{u}(n)$ in the place of $u(n)$, the third order cross-cumulants can be calculated and ARMA coefficients can be estimated.

\section{RTESULTS AND DISCUSSIONS}

Simulation studies have been performed to examine the proposed ARMA model coefficients estimation using the QRD-TOC algorithm. Several experiments were simulated at different signal-to-noise ratio (SNR) on the output sequence. A zero-mean, independent and identically distributed (i.i.d), and exponentially distributed non-Gaussian process was generated at the input. The results of the proposed method were compared with well-known procedures such as the Residual Time Series (RTS) and the Q-slice algorithm (QSA) methods at different levels of SNR on the output. The commands armarts and armaqs were used from the Higher-Order Spectral Analysis Toolbox User's Guide [20] to estimate the ARMA coefficients using the RTS and the QSA methods, respectively. All simulations were implemented and taken as the average of 100 Monte Carlo runs. The computations were performed in MATLAB tools. The data length of the sequence was taken to be $N=1500$ points for each experiment.

Example 1. Data were drawn using the $\operatorname{ARMA}(2,2)$ model

$$
\begin{gathered}
y(n)+0.3 y(n-1)+0.25 y(n-2)=u(n)+0.95 \\
u(n-1)+0.65 u(n-2)
\end{gathered}
$$

This is an ARMA $(2,2)$. It has two poles and two zeros. The poles are located at $-0.15 \pm j 0.477$. The zeros are located at $0.475 \pm j 0.6514$. The sequence $y(n)$ is observed in additive Gaussian noise $s(n)=y(n)+w(n)$. A zero-mean, nonGaussian distribution (namely exponential distribution) was used to generate the input sequence. Then, the input signal was driven through the system in (41). The output sequence was corrupted with additive Gaussian noise at SNR of $10 \mathrm{~dB}$ on the output sequence. The, the cumulant matrix $C_{p q}$ in (15) was built. The method in $[18,19]$ was used to estimate the input sequence. The experiment estimated the ARMA coefficients using the RTS, the QSA, and the proposed QRD-TOC methods. Table I presents the results of 100 Monte Carlo simulations for the RTS, QSA, and the proposed QRD-TOC algorithms at SNR of $10 \mathrm{~dB}$.

Table 1. True and estimated ARMA $(2,2)$ model coefficients in Example 1, $a(0)=1, b(0)=1$.

\begin{tabular}{|c|c|c|c|c|}
\hline & True & RTS & QSA & $\begin{array}{c}\text { QRD- } \\
\text { TOC }\end{array}$ \\
\hline $\mathrm{a}(1)$ & 0.3 & 0.2560 & -0.5811 & 0.2984 \\
\hline $\mathrm{a}(2)$ & 0.25 & 0.2608 & 0.2751 & 0.2456 \\
\hline $\mathrm{b}(1)$ & 0.95 & 1.0012 & 0.7311 & 0.9505 \\
\hline $\mathrm{b}(2)$ & 0.65 & 0.6196 & 1.3022 & 0.6464 \\
\hline
\end{tabular}


Example 2. Data were drawn using the $\operatorname{ARMA}(6,4)$ model

$$
\begin{aligned}
& y(n)+0.7907 y(n-1)+0.042 y(n-2)-0.556 y(n-3) \\
& -0.0247 y(n-4)+0.385 y(n-5)+0.303 y(n-6)=u(n) \\
& +0.345 u(n-1)+0.53 u(n-2)+0.399 u(n-3)+ \\
& 0.814 u(n-4)
\end{aligned}
$$

This model has six poles and four zeros, ARMA $(6,4)$. The poles are located at $0.7102 \pm j 041,-0.43 \pm j 0.7448$, and $0.6755 \pm j 0.39$. The zeros are located at $0.485 \pm j 0.84$, $0.6576 \pm j 0.6576$.

The data was generated as in Example 1. The same procedure was followed in estimating the ARMA coefficients. The experiment estimated the ARMA coefficients using the RTS, the QSA, and the proposed QRD-TOC methods. Table II presents the results of 100 Monte Carlo simulations for the RTS, QSA, and the proposed QRD-TOC algorithms at SNR of $20 \mathrm{~dB}$.

Table 2. True and estimated ARMA $(6,4)$ model coefficients in Example 2, $a(0)=1, b(0)=1$.

\begin{tabular}{|c|c|c|c|c|}
\hline & True & RTS & QSA & $\begin{array}{c}\text { QRD- } \\
\text { TOC }\end{array}$ \\
\hline $\mathrm{a}(1)$ & 0.7907 & 0.7350 & 0.7023 & 0.7941 \\
\hline $\mathrm{a}(2)$ & 0.0420 & -0.0010 & -0.0409 & 0.0548 \\
\hline $\mathrm{a}(3)$ & -0.5556 & -0.5268 & -0.5884 & -0.5658 \\
\hline $\mathrm{a}(4)$ & -0.0247 & -0.0220 & 0.0231 & -0.0333 \\
\hline $\mathrm{a}(5)$ & 0.3846 & 0.3793 & 0.3772 & 0.3955 \\
\hline $\mathrm{a}(6)$ & 0.3026 & 0.3071 & 0.2822 & 0.3106 \\
\hline $\mathrm{b}(1)$ & 0.3452 & 0.3257 & 0.1998 & 0.3588 \\
\hline $\mathrm{b}(2)$ & 0.5300 & 0.5531 & 0.6127 & 0.5352 \\
\hline $\mathrm{b}(3)$ & 0.3985 & 0.3917 & 0.5432 & 0.3654 \\
\hline $\mathrm{b}(4)$ & 0.8138 & 0.7199 & 0.9914 & 0.8023 \\
\hline
\end{tabular}

Example 3. (Colored Gaussian) Consider the ARMA $(6,4)$ process generated by the difference equation in (42). The colored Gaussian noise $w(n)$ was generated as a sinc function:

$$
h(n)=0.3 \operatorname{sinc}(0.01 n)
$$

The experiment estimated the ARMA coefficients using the RTS, the QSA, and the proposed QRD-TOC methods. Table III presents the results of 100 Monte Carlo simulations for the RTS, QSA, and the proposed QRD-TOC algorithms at SNR of $20 \mathrm{~dB}$.

Table 3. True and estimated ARMA $(6,4)$ model coefficients in Example 3, $a(0)=1, b(0)=1$, colored.

\begin{tabular}{|l|l|l|l|l|}
\hline & True & RTS & QSA & $\begin{array}{l}\text { QRD- } \\
\text { TOC }\end{array}$ \\
\hline $\mathrm{a}(1)$ & 0.7907 & 0.7282 & 0.7425 & 0.7840 \\
\hline $\mathrm{a}(2)$ & 0.0420 & -0.0012 & -0.0124 & 0.0343 \\
\hline $\mathrm{a}(3)$ & -0.5556 & -0.5167 & -0.5688 & -0.5608 \\
\hline $\mathrm{a}(4)$ & -0.0247 & -0.0267 & 0.0016 & -0.0296 \\
\hline $\mathrm{a}(5)$ & 0.3846 & 0.3708 & 0.3687 & 0.3960 \\
\hline
\end{tabular}

\begin{tabular}{|l|l|l|l|l|}
\hline $\mathrm{a}(6)$ & 0.3026 & 0.3030 & 0.2835 & 0.3142 \\
\hline $\mathrm{b}(1)$ & 0.3452 & 0.3249 & 0.3681 & 0.3450 \\
\hline $\mathrm{b}(2)$ & 0.5300 & 0.5419 & 0.5114 & 0.5115 \\
\hline $\mathrm{b}(3)$ & 0.3985 & 0.3997 & 0.3055 & 0.3648 \\
\hline $\mathrm{b}(4)$ & 0.8138 & 0.7034 & 0.5581 & 0.7639 \\
\hline
\end{tabular}

\section{CONCLUSION}

The paper developed an efficient procedure for calculating ARMA model coefficients using QRD-TOC from the available output data. The results of the simulated experiments demonstrate the good performance of the developed technique when the observed sequence is contaminated by additive Gaussian noise. The proposed technique was compared with known procedures such as RTS, and the QSA. As it can be seen from Tables I, II, and III, the proposed QRD-TOC technique is more accurate than the RTS and QSA methods.

\section{REFERENCES}

[1] A. Al-Smadi, "Cumulant-based order selection of nonGaussian autoregressive moving average models: the corner method", Signal Processing, vol. 85, no. 3, pp. 449-456, 2005.

[2] G.B. Giannakis and J.M. Mendel, Identification of nonminimum phase systems using higher order statistics, IEEE Trans. Acoust., Speech, Signal processing, vol. 37, Issue: 3, pp. 360-377, 1989.

[3] A. Swami and J. Mendel, ARMA parameters estimation using only output cumulants, IEEE Trans. Signal Processing, vol. 38, no. 7, pp. 1257-1265, 1990.

[4] G.B. Giannakis, J.M. Mendel, Cumulant-based order determination of non-Gaussian ARMA models, IEEE Trans. Acoust. Speech Signal Process. 38, Issue: 8, 1411-1423, 1990.

[5] S. U. Pillai, T.I. Shim, D.C. Youla, A new technique for ARMA-system identification and rational approximation, IEEE Trans. Signal Process, vol. 41, no. 3, March 1993, 1281-1304.

[6] A. Al-Smadi and M. Smadi, "Study of the reliability of a binary symmetric channel under non-Gaussian disturbances," International Journal of Communication Systems, vol. 16. no. 10, pp. 865-973, 2003. DOI: 10.1002/dac.626

[7] A. Al-Smadi and D.M. Wilkes, "Robust and accurate ARX and ARMA model order estimation of non-Gaussian processes", IEEE Trans. Signal Processing, vol. 50, no.3, pp. 759-763, 2002.

[8] A. Al-Smadi, "Automatic Identification of ARMA Processes, " International Journal of General Systems, vol. 38, no. 1, pp.29-41, 2009.

[9] Ali Amiri and Mahmood Fathy, " Video Shot Boundary Detection Using QR-Decomposition and Gaussian Transition Detection," EURASIP Journal on Advances in Signal Processing, vol. 2009.

[10] Thitapa Poomvichid, Pantida Patirupanusara, and Mahasak Ketcham, " The QR Code for Audio 
Watermarking using Genetic Algorithm," International Conference on Machine Learning and Computer Science (IMLCS'2012), August 11-12, pp. 171-174, 2012.

[11] Junxiang Wang, Ying Liu, and Yonghong Zhu, "Color Image Blind Watermarking Algorithm Based on QR Decomposition and Voting in DWT Domain," International Journal of Security and Its Applications, vol. 10, no. 11, pp.33-46, 2016. DOI: 10.14257/ijsia.2016.10.11.04

[12]E. Anderson, Z. Bai, and J. Dongarra, "The Generalized QR Factorization and its Applications," Linear Algebra and its Applications, vol. 162-164, February 1992, pp. 243-271.

[13]J.Mehena, L.Swain, and G.Patnaik, " System Identification Based on QR-Decomposition," Int. J. of Intelligent Computing and Applied Sciences, vol. 1, Issue. 1, pp 31-40, 2013.

[14]R. Pickhardt and H. Unbehauen, " A robust multi-step least squares identification method based on pseudorecursive QR-factorization technique," IFAC System Identification, vol. 27, Issue 8, pp. 1435-1440, 1994.

[15] T.K. Moon and W. C. Stirling, Mathematical Methods and Algorithms for Signal Processing, Prentice Hall, Saddle River, NJ, 2000.

[16] Todd K. Moon and Wynn C. Stirling, Mathematical Methods and Algorithms for Signal Processing, Prentice Hall, NJ, 2000.

[17]L. Scharf, Statistical Signal Processing: Detection, Estimation, and Time Series Analysis, Addison-Wesley Publishing Company, New York, 1991.

[18] J. Durbin, "The fitting of time series models," Review of the International Statistical Institute, vol. 28, no. 3, pp. 233-243,1960. DOI: 10.2307/1401322.

[19] A. Al-Smadi and A. Al-Zaben, "ARMA Model Order Determination Using Edge Detection: A New Perspective," Circuits, Systems \& Signal Processing (CSSP), Springer, vol. 24, no. 6, pp. 723-732, November 2005.

[20]A. Swami, J. M. Mendel, and C. Nikias, Higher order spectral an analysis Toolbox-User's Guide, The MathWorks, Inc., Natick, MA, 1998.
Creative Commons Attribution License $\mathbf{4 . 0}$ (Attribution 4.0 International, CC BY 4.0)

This article is published under the terms of the Creative Commons Attribution License 4.0

https://creativecommons.org/licenses/by/4.0/deed.en_US 